\title{
On the Actuarial Simulation of the General Pareto Distribution of Catastrophe Loss
}

\author{
Xiaojun Pan, Chengyi Pu \\ Economic College, Southwest University for Nationalities, ChengDu 610041, China \\ 巨灾损失分布的广义 Pareto 精算模拟分析
}

潘小军, 蒲成毅

西南民族大学经济学院, 成都 610041, 中国

\begin{abstract}
Precisely estimation of Cat-loss is not only the foundation of risk analysis, but also the premise of product design and the practice of insurance compensation. The law of large numbers generally assumes risks have normal distributions, which reduces the accuracy of damage assessment and influences the pricing of cat-insurance due to negligence of the extreme value at both sides of the distribution. Data of more than 100 million Yuan of earthquake disaster loss from 1969-2014 presents the characteristics of right skewed peak, excess kurtosis and heavy tail. Furthermore, the comparison of QQ plot, parameter estimation as well as test of model parameters between $\mathrm{N}$-distribution, E-distribution, W-distribution and P-distribution shows that the generalized Pareto distribution fits the earthquake loss perfectly, and significantly improves the estimate precision.
\end{abstract}

Keywords: The General Pareto Distribution;

Catastrophe Loss; Actuarial Simulation

\section{摘要}

损失的精准估算是巨灾风险分析的基础。保险经 营的大数法则通常假定风险标的服从高斯分布, 对位于损失分布两端尾部极值损失数据忽略的拟
合建模, 既导致风险分析严重失真, 更影响损失 评估精度与巨灾保险产品费率厘定。本文选取我 国 1969-2014 年间直接经济损失额超过 1 亿元的 104 次地震灾害损失数据的分析发现, 分布呈现严 重的右偏和尖峰厚尾特征, 另外, 数据的 Pareto、 Normal、Exponential、Weibull 等分布的 QQ 图比 较, 以及几种分布模型参数估计与检验的比较表 明, 地震直接经济损失数据的广义 Pareto 分布模 型的拟合效果最恰当, 可显著提高巨灾风险损失 的估计精度, 是提升政府减灾能力和保障商业保 险产品设计与灾害赔偿的前提。

关键词: 巨灾损失分布; QQ 图; GPareto 拟合

\section{1. 前言}

全球巨灾近年来呈频发和并发态势，大量 人员伤亡和严重经济损失呈递增趋势, 其中地 震、洪水、台风、暴风雨和旱灾等重大灾害造 成的经济损失尤其巨大 (如图 1、表 1)。这种 趋势促进了巨灾保险及其衍生品设计的创新, 以及巨灾保险与灾害经济学、灾害社会心理等 学科的融合发展, 巨灾保险已成重要的市场化

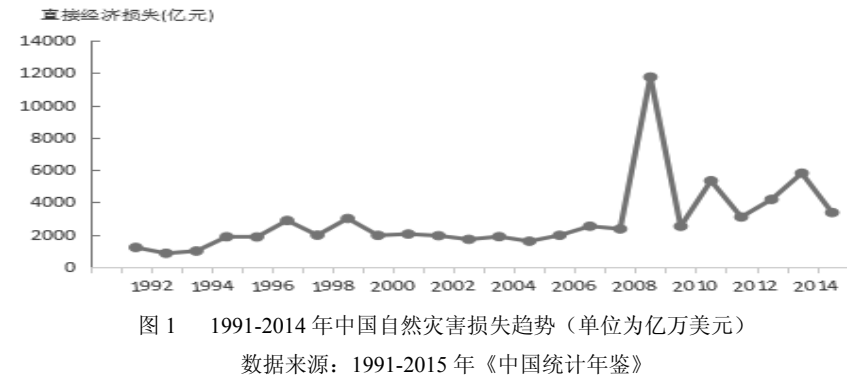


Risk Analysis and Crisis Response in Big Data Era (RAC-16)

表 1. 1969-2015 年我国地震损失极值数据的统计描述

\begin{tabular}{ccccc|cc}
\hline 极值数据分组 (亿元) & 频数次 & 频率\% & 累计频率 & 频率密度 & \multicolumn{2}{|c}{ 极值数据描述性统计 } \\
\hline $\mathbf{1 - 1 0}$ & 68 & 65.38 & & 0.65 & 均值 & 106.07 \\
$\mathbf{1 0 - 2 0}$ & 15 & 14.42 & 79.81 & 0.14 & 中位数 & 4.72 \\
$\mathbf{2 0 - 3 0}$ & 6 & 5.77 & 85.58 & 0.06 & $25 \%$ 分位数 & 2.12 \\
$\mathbf{3 0 - 4 0}$ & 2 & 1.92 & 87.50 & 0.02 & $50 \%$ 分位数 & 3.71 \\
$\mathbf{4 0 - 5 0}$ & 4 & 3.85 & 91.35 & 0.04 & $75 \%$ 分位数 & 11.73 \\
$\mathbf{5 0 - 6 0}$ & 2 & 1.92 & 93.27 & 0.02 & 标准差 & 792.09 \\
$\mathbf{6 0 - 7 0}$ & 0 & 0.00 & 93.27 & 0.00 & 最大值 & 8048.16 \\
$\mathbf{7 0 - 8 0}$ & 1 & 0.96 & 94.23 & 0.01 & 最小值 & 1.06 \\
$\mathbf{8 0 - 9 0}$ & 0 & 0.00 & 94.23 & 0.00 & 偏度 & 9.83 \\
$\mathbf{9 0 - 1 0 0}$ & 0 & 0.00 & 94.23 & 0.00 & 峰度 & 80.82 \\
$\mathbf{1 0 0}$ 亿以上 & 6 & 5.77 & 100.00 & 0.06 & & \\
\hline
\end{tabular}

数据来源: 历年的《中国地震年鉴》整理。

防灾减灾工具(SwissRe,2011) ${ }^{[1]}$ 。然而, 巨灾风 险分析一直滞后, 保险经营的大数法则通常假 定风险标的服从高斯分布，而对位于分布两端 尾部的巨灾损失作如此拟合建模既导致风险分 析的严重失真, 影响了损失评估精度, 制约了 政府防灾减灾能力建设与巨灾保险产品设计能 力的提升。本文希望通过对超过一定阀值的巨 灾直接经济损失额尾部数据进行建模和损失额 的极值分位数估计, 夯实巨灾风险分析与巨灾 保险产品设计的基础。

\section{2. 国内外文献综述}

在自然灾害损失或金融风险的整体分布拟 合分析中, 常常假设随机变量服从 Gamma、

Normal、Exponential、Weibull 等分布，这种简 易拟合因对巨灾损失的偏峰厚尾分布特征和尾 部极端数据的忽略, 往往会导致分析失真。 20 世纪初, 德国统计学家 Bortkiewicz (1922) ${ }^{[3]}$ 研究了来自正态样本最大值和最小值的分布规 律, 首次提出了极值概念。随着极端值分析的 出现, Fisher 与 Tippet (1928) 提出了极值分布 模型的三种标准形式: Frechet、Weibull 及 Gumbel 分布 ${ }^{[4]}$ 。随机样本以及随机过程中极端 情况的统计规律的初步应用推动了极值理论的 不断完善。Jenkinson (1955) 从风险研究提出 广义极值分布 (GEV) ${ }^{[5]}$ 。Mandelbrot (1963) 证实金融资产收益率不服从正态分布, 而服从 厚尾分布 ${ }^{[6]}$ 。Haan (1970) 发现超过门阀值的数 据服从广义帕累托分布 ${ }^{[7]}$ 。Smith（1986）用广 义帕累托分布（GPD）基于观测值中所有超过 阀值的部分构建更加完善的 POT (Peaks over threshold）模型 ${ }^{[8]}$ 。McNeil（1999）不仅对极值 理论进行了总结和评述 ${ }^{[9]}$, 还与 Frey (2000) 用
极值分析方法研究了异方差的金融时间序列与 尾部相关的风险测量和模型估计 ${ }^{[10]}$ 。Pickands （1975）基于经典 Pareto 分布（PD）拓展的广 义 Pareto 分布 (GPD) 后, 对于洪水、风力、 气温、降雨等自然现象的分析预测发挥着重要 作用, 甚至在金融保险精算领域的极端现象分 析也优势凸显 ${ }^{[11]}$ 。比如, 股票交易量与收益率 的非正态厚尾特征, 保险标的损失数据的非负 偏厚尾特征, 应用 GPD 模型均可以很好地拟合 这些数据并预测最大损失 (Beiriant, Teugels, Vynckier,1996) ${ }^{[12]}$ 。但是, 基于 GPD 的 POT 模 型参数估计和阈值的适当确定, 对保证超过某 阈值的数据近似服从 GPD 成为应用 POT 模型进 行拟合与计算的关键和难点。

国内极值理论研究起步较晚。极值理论计 算 VaR 准确性提高（田宏伟等, 2000） ${ }^{[13]}$, 基 于极值理论的完全参数方法改进和 POT 模型的 传统阈值选择 hill 估计方法改进 (王元月等, 2012) 提高了估算精度 ${ }^{[14]}$ 。其次, 资本市场收 益率的极值理论分析实证, 以及与方差协方差 方法 (周开国, 2002) ${ }^{[15]}$ 和正态分布等模拟方 法的比较分析 (欧阳资生, 2008) ${ }^{[16]}$, 甚至结 合 ARMA-AGARCH 模型的股票收益率尾部特 征分析表明极值理论在金融时间序列数据分析 中很有优势。另外, 杨刚等 (2008) ${ }^{[17]}$ 用指数 回归模型构造 Hall 分布族的极值分位数估计对 火灾保险的巨额索赔数据拟合。肖海清等 (2013) ${ }^{[18]}$ 以我国洪水损失数据极值分析得到 洪水再保险保费。刘鹃等 (2009) ${ }^{[19]}$ 对我国地 震损失额的对数正态分布拟合构建地震巨灾债 券的定价模型。卓志等 (2011) ${ }^{[20]}$ 通过巨灾风 险厚尾分布的比较分析 POT 模型及其应用优 势。钱小仕等 (2012) ${ }^{[21]}$ 台湾地区地震历史资 
料的广义极值分布得出地震震级次数的理论值 与实际值完全一致的结论。

综上所述，尽管 Gamma、Normal、 Exponential、Weibull 等分布在拟合巨灾风险的 整体分布有简便优势, 由于对巨灾损失分布的 尾部数据严重忽略, 导致重要信息漏损, 相反 基于 GPD 的 POT 模型在拟合厚尾分布时则克服 了传统模拟的不足, 更精准地刻画了巨灾损失 分布的尾部极端值所包含的信息。但是, 基于 GPD 的 POT 模型参数估计和阈值的适当确定则 成为极值分析应用 POT 模型进行拟合与计算的 关键和难点。尽管风险分析还有许多困惑, 但 极值分析对巨灾损失的拟合效果与分析预测的 准确性都是公认的 ${ }^{[22]}$ 。因此, 本文以 1969-2014 年我国直接经济损失额超过 1 亿元的 104 次地 震灾害损失数据样本, 通过 Gamma、Normal、 Exponential、Weibull 等各种损失分布拟合的 QQ 图比较, 以及基于广义 Pareto 分布的 POT 模型 参数估计和阈值的适当确定, 以保证超过某阈 值的数据近似服从 GPD 分布, 提高巨灾风险损 失的估算精度。

本文的结构安排是：在巨灾风险分析现实 困境和前人研究缺陷基础上确立研究主题; 以 我国历年重特大地震灾害损失数据为样本, 通 过极端阀值数据选取设定, 寻找地震巨灾损失 数据的统计性规律, 并对超过一定阀值的巨灾 直接经济损失额的尾部数据进行建模比较, 以 及损失额的极值分位数拟合估计, 确定拟合效 果最好的分布形式, 提出实现巨灾风险损失估 算精度的建议。

\section{3. 震损失极值数据阀值的设定}

到底多大的地震灾害损失才算巨灾? 目前 国际保险界对此没有统一的定义 ${ }^{[23]}$, 而基于 GPD 的 POT 模型数据阈值的适当确定是极值分 析拟合的关键, 极值数据阀值的设定一直是难 点。

美国保险服务局 (ISO) 财产理赔部（1998） 定义: 至少造成 2500 万美元 (1998 年的价格) 财产损失, 并影响到大部分的保险人和被保险 人的事件。标准普尔 (1999) 巨灾损失定义: 一个或一系列的风险事件导致的保险损失超过 500 万美元。国际经济合作组织 (OECD) (2003) 定义: 造成大量人员伤亡, 财产损失和大面积 的基础设施被破坏, 灾区政府无力控制, 要借 助于国际国内的通力合作才能应对的事件。
Chichilnisky（2000） ${ }^{[24]}$ 和 Hansson et al (2008) ${ }^{[25]}$ 定义巨灾风险为诸如洪水、地震、暴风雨、 干旱等自然灾害导致重大损失的极端小概率事 件。GAO (美国总审计署) (2002) ${ }^{[26]}$ 认为巨灾 就是能够导致严重经济损失的罕见事件。

Mohamed Gad-el-Hak (2008) ${ }^{[27]}$ 定义巨灾为死 亡 10000 人以上或受灾面积大于 $1000 \mathrm{~km}^{2}$ 的灾 害事件。SwissRe (2009) ${ }^{[28]}$ 定义: 轮船损失为 1752 万美元; 航空损失为 3440 万美元; 其他损 失 4270 万美元; 或总损失为 8540 万美元为巨 灾损失。从人员伤亡的角度定义: 死亡或失踪 20 人以上; 受伤 50 人以上; 无家可归 2000 人 以上为巨灾损失。日本在地震保险经营中将超 过 750 亿日元的损失定义为巨灾。另外, 国内 的马宗晋 (1990) ${ }^{[29]}$ 定义死亡人数 10000 人以 上, 直接经济损失 10 亿元以上的灾害损失为巨 灾。汤爱平 (1999) ${ }^{[30]}$ 按国家省 (市) 县三级 的损失占 GDP 比重定义巨灾为国家级灾害损失 占 GDP 比值大于 $0.002 \%$, 伤亡比例大于 $0.08 \%$ 的事件; 省市级灾害损失占本区 GDP 比值大于 $1 \%$, 伤亡比例大于 $0.005 \%$ 的事件; 县级灾害损 失占本区 GDP 比值大于 $0.3 \%$, 伤亡比例大于 $2 \%$ 的事件。李军 (2005) ${ }^{[31]}$ 将巨灾定义为导致 不同风险单位之间的损失具有相关性且使保险 失灵的风险。邓国取（2009） ${ }^{[32]}$ 定义巨灾为一 定时期内（通常为 1 年）小概率且损失大于预 期, 累计损失超过主体承受能力的事件。马宗 晋 (2010) ${ }^{[33]}$ 修正巨灾定义为死亡人数 10000 人以上, 直接经济损失 1000 亿元以上的特大灾 害损失。史培军等 $(2010)^{[34]}$ 定义巨灾为百年 一遇造成大量人员伤亡和财产损失, 须借助外 力才能应对和恢复的重大灾害事件。

显然, 巨灾是一个相对概念, 应相对于主 体的承受能力来确定极值数据的阀值。中国人 均 GDP 较低, 人们承受风险的能力较差, 据此 国情，本文对巨灾风险的定义是; 损失超过 1 亿人民币, 伤亡 200 人以上, 影响到大面积的 被保险人的自然灾害和人为损失事件。

\section{4. 地震损失极值数据的统计描述}

本文选取 1969-2015 年我国直接经济损失 超过 1 亿元的 104 次地震灾害损失数据样本, 来源于历年的《中国地震年鉴》, 经过价格调整 (以 2005 年的价格为标准) 整理得出, 数据分 组得到各组频数、频率分布、频率密度 (表 2), 从表 2 中可以看出损失额在 1-10 亿元组的损失 
Risk Analysis and Crisis Response in Big Data Era (RAC-16)

表 2. 地震直接经济损失的 6 种概率分布拟合与损失次数拟合

\begin{tabular}{cccc|ccc}
\hline 分布名称 & & 参数值 & $\mathbf{K S}$ 检验 $\boldsymbol{p}$ 值 & 次数 $\boldsymbol{n}$ & 频数 & Poisson 拟合值 \\
\hline 广义帕累托分布 & $\lambda=1.0553$ & $\beta=0.5950$ & 0.7658 & 0 & 11 & 9 \\
咖马分布 & $\alpha=0.8540$ & $\beta=73.2212$ & 0.6558 & 1 & 5 & 4 \\
对数正态分布 & $\mu=3.4503$ & $\theta=1.4743$ & 0 & 2 & 4 & 3 \\
指数分布 & $\lambda=0.1162$ & & 0.2743 & 3 & 3 & 1 \\
威布尔分布 & $\gamma=0.4457$ & $\mathrm{c}=13.3454$ & 0.0345 & 4 & 2 & 1 \\
正态分布 & $\mu=115.8355$ & $\beta=884.5950$ & 0.1327 & 5 & 1 & 1 \\
\hline
\end{tabular}

频率最大为 $65.38 \%, 100$ 亿以上组的损失频率 5.77\%与 20-30 组的损失频率相等。另外, 损失 额的 $25 \%$ 分位数与 $50 \%$ 分位数相差不大, 但 $75 \%$ 分位数与 $50 \%$ 分位数相差较大; 由于有损失额 相当大的数据 (如 2008 年汶川地震的损失直接 经济额为 8523 亿元), 其最大值是最小值的 8000 多倍; 偏度系数达 9.83 , 峰度为 80.82 , 损失数 据严重右偏, 存在明显的尖峰厚尾的特征。从 损失额频数分布曲线图 (图 2) 和地震损失额分
布曲线图 (图 3), 也都可以看出损失分布呈严 重右偏, 具有明显的厚尾分布特征。

\section{5. 地震损失极值数据分布 $\mathrm{QQ}$ 图比较}

用 Eviews5.0 做出 Normal、Exponential、 Weibull 和 Pareto 等分布的 QQ 图（图 4、图 5、 图 6、图 7), 通过比较可知, Normal 分布不能 很好的拟合巨灾损失的尾部特征，而 Exponential 和 Weibull 分布的拟合较好, 用 Pareto 分布则能更好的拟合极值损失数据。

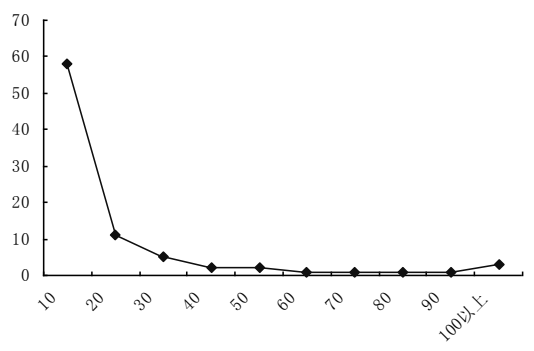

图 2. 地震损失额频数分布曲线图

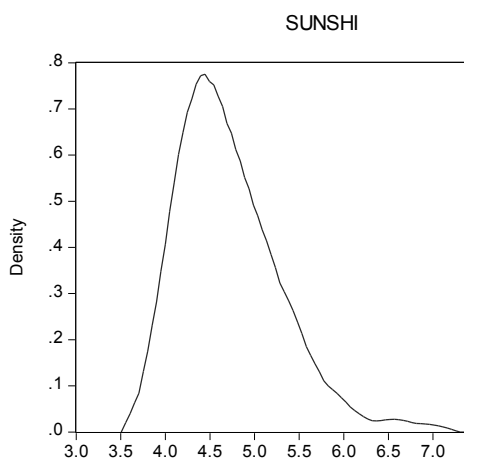

图 3. 地震损失额分布曲线图 
Risk Analysis and Crisis Response in Big Data Era (RAC-16)

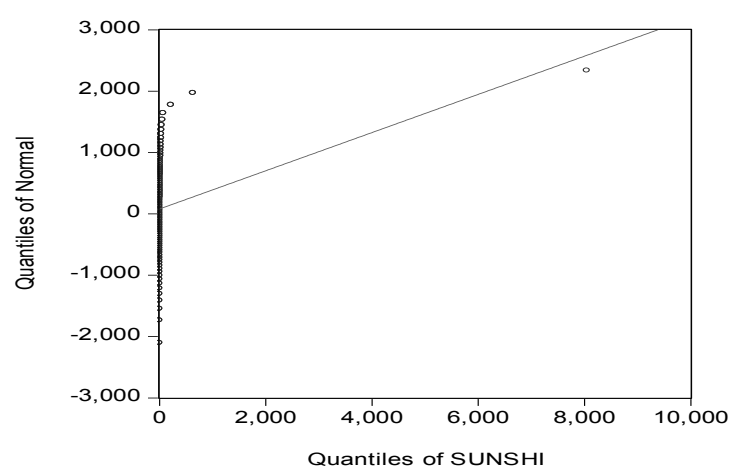

Figure 4. Diagram QQ of Normal Distribution

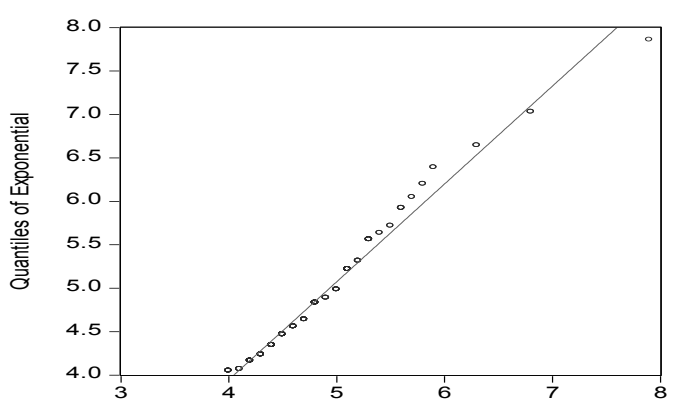

Quantiles of SUNSHI

Figure 5. Diagram QQ of Exponential Distribution

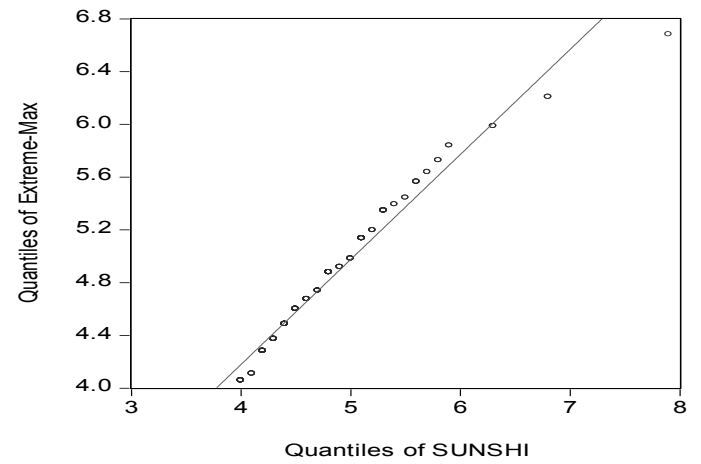

Figure 6. Diagram QQ of Weibull Distribution 
Risk Analysis and Crisis Response in Big Data Era (RAC-16)

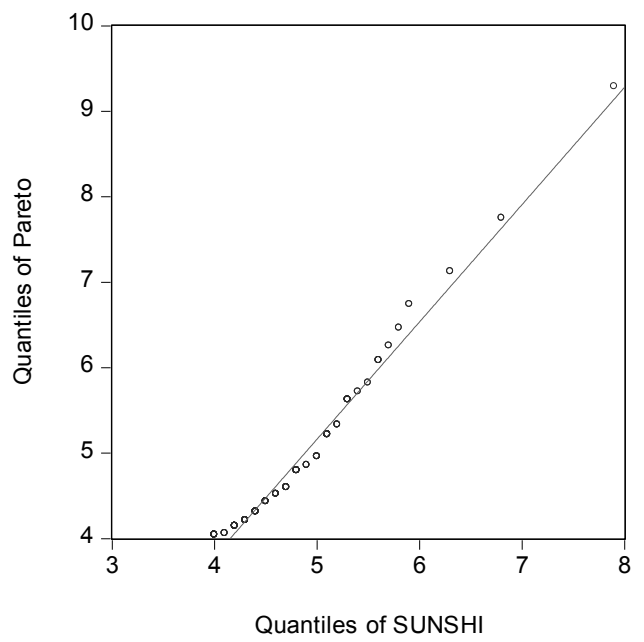

Figure 7. Diagram QQ of Pareto Distribution.

6. 地震损失极值数据分布拟合比较 6.1 极值数据的各种分布拟合比较

对极值数据采用极大似然法分别估计帕累 托分布、伽马分布、正态分布、对数正态分布、 指数分布、威布尔分布等概率分布函数的参数, 用 K-S 检验法检验其拟合优度, 通过比较确定 合适的概率分布函数。从表 3 可知, Pareto 拟合 优度检验效果最好。 K-S 检验的 P 值为 0.7658 大于 $5 \%$ 显著性水平, 因此, 确定广义帕累托 分布为地震巨灾直接经济损失额极值数据的统 计分布 (见图 7)。

\section{2 极值数据的广义 Pareto 分布拟合}

广义 Pareto 分布 (Generalized Pareto Distribution, 简称 GPD) 是对传统 Pareto 分布 的改进。因为, 尽管传统 Pareto 分布对随机现 象的尾部特征具有良好的模拟性, 但是, Pareto 分布的概率密度函数为:

$$
\begin{aligned}
& \rho_{X}(x)=\beta \gamma^{\beta} / x^{(\beta+1)} \\
& \gamma>0, \beta>0, x \geq \gamma, \beta>0 \text { 为形状参数, }
\end{aligned}
$$

$\gamma>0$ 为门槛参数, 对数形式为:

$$
\ln (1-F(x))=\beta \ln r-\beta \ln x .
$$

显然, 样本观测点在双对数坐标图中 Pareto 分布是一斜率为 $\beta$ 的直线, 这一线性关系是判 断一组样本是否满足 Pareto 分布的依据, 也是 根据此性质利用最小二乘法来确定门槛值的, 不过, 这种方法带有一定的主观性。改进的广 义 Pareto 分布则能克服此缺陷。本文采用 1969-2011 年间 43 年中我国地震直接经济损失 超过 1 亿元损失的数据 (共 83 次) 作为极值样 本观测数据, 讨论大于阀值的观测值的分布。

随机变量 $X$ 超过阀值 $\mu$ 的条件分布函数:

$$
F_{\mu}(y)=p\{X-\mu \leq y \mid X>\mu\}, X \geq 0
$$

$Y=X-\mu$ 为超出损失, 据条件概率有:

$$
\begin{aligned}
& F_{\mu}(y)=\frac{F(\mu+y)-F(\mu)}{1-F(\mu)} \\
& F(x)=F_{\mu}(x-\mu)(1-F(\mu))+F(\mu)
\end{aligned}
$$

称 $e(\mu)=E(X-\mu \mid X>\mu)$ 为随机变量的 平均超出量函数。

若随机变量有以下的分布函数: 
Risk Analysis and Crisis Response in Big Data Era (RAC-16)

$$
\begin{aligned}
& F(x, \mu, \sigma, \delta)=\left\{\begin{array}{l}
1-\left(1+\beta \frac{x-\mu}{\lambda}\right)^{-1 / \delta}, \beta \neq 0 \\
1-\exp \left(\frac{x-\mu}{\lambda}\right), \beta=0
\end{array}\right. \\
& x \geq \mu, 1+\beta \frac{x-\mu}{\lambda}>0
\end{aligned}
$$

则随机变量X服从三参数广义Pareto分布。其

中, $\mu$ 位置参数、 $\lambda$ 刻度参数、 $\delta$ 形状参数。

另外, GPD的参数用最大似然法估计得到。

设 $\mathrm{X}_{1}, \mathrm{X}_{2 \ldots \ldots} \mathrm{X}_{\mathrm{n}}$ 为来自 $p(\gamma, \beta)$, 则 $\gamma, \beta$ 的极大
尾部数据进行建模和损失额极值分位数的估计 成为极值分析的重要内容。本文通过地震损失 极值数据的各种概率分布的比较, 发现用广义 帕累托分布可以准确地描述损失分布尾部的分 位数特征, 实际拟合的效果较好, 而且, 具有 良好的函数解析形式, 明显提高了巨灾风险分 析精度, 奠定了巨灾保险产品设计的科学性。

\section{Acknowledgements}

This study was supported by special Programs of the central university (2014SZYTD01) ; National Social Science Foundation of China (No.12XJL012); and Chinese post doctoral fund (2011M501409、 2013T60851).

似然估计值为:

$$
\hat{\lambda}=\min _{1 \leq i \leq n} x_{i}=x_{n}^{(1)} \hat{\beta}=n\left[\sum_{i=1}^{n} \ln \frac{x_{i}}{x_{n}^{(1)}}\right]^{-1}
$$

门槛值为:

$$
U=(9 n-18)^{1 / 2}\left[T(2 n-4)^{1 / 3}+1 /(9 n-18)-1\right]
$$

$\mathrm{n}$ 为样本参数个数, $\mathrm{T}$ 为拟合检验统计量。

把 83 个样本数据代入表达式计算得到:

$$
\hat{\gamma}=1.0553, \hat{\beta}=0.5950
$$

则地震损失极值广义 Pareto 分布为:

$$
\begin{aligned}
& F(x)=1-\left(1+\beta \frac{x-\mu}{\lambda}\right)^{-1 / \beta} \\
& =1-\left(1+0.5950 \frac{x-\mu}{1.0553}\right)^{-1 / 0.5950}
\end{aligned}
$$

\section{3 极值数据的损失次数拟合}

设地震损失次数服从参数 $\lambda$ 的泊松分布:

$$
p\{\xi=k\}=\frac{\lambda^{k}}{k !} e^{-\lambda}, k=0,1,2 \ldots \ldots \ldots
$$

运用矩估计 $\hat{\lambda}=E Y$, 根据表 2 中我国地震 直接经济损失额的极值样本数据, 计算可得样 本的一阶原点矩 $\bar{x}=115.84$

运用参数 $\lambda=115.84$ 的泊松分布对地震损 失的次数进行拟合 (表 2)。

另外，按上述拟合的泊松分布，每一年发 生 $\mathrm{i}$ 次损失额度超过 1 亿元的概率:

$P_{i}(i=1,2,3,4,5,6)$ 分别为 $0.44,0.20$, $0.16,0.12,0.08,0.04$ 。

\section{7. 结论}

巨灾损失具有典型的右偏和尖峰厚尾特 性。如果采用常用的正态分布建模, 则位于分 布两端的损失极端值容易被忽略, 影响分析结 论的精度。如何对巨灾直接经济损失额分布的

本课题受到中央高校资助项目 （2014SZYTD01）、国家社科基金（12XJL012）、中 国博士后基金（2011M501409、2013T60851）等项 目基金的资助。

\section{参考文献}

[1] Swiss Re. (2011), Natural catastrophes and man-made disasters in 2010: a year of devastating and costly events.

[2] 地震直接经济损失数据: http: / / www.chinadisaster. $\mathrm{cn} /[\mathrm{DB} / \mathrm{OL}]$.国家地震科学数据共享 中心, 2013

[3] L.Von Bortkiewicz (1922), Variationsbreite und mittlerer Fehler. Berlin Math. Ges. Sitzungsber. 21: 3- 11.

[4] R.A.Fisher, L.H.C.Tippett (1928), Limiting forms of the frequency distribution of the largest or smallest member of a sample. Proceedings of the Cambridge Philosophical Society.24:180-190.

[5] A.F. Jenkinson (1955), The frequency distribution of the annual maximum(or minimum) values of meteorological elements, Quarterly Journal of the Royal Meteorological Scoiety,87:158-171.

[6] B. Mandelbrot (1963a), New methods in statistical economics, Journal of Political Economy, 71:421-440.

[7] D.L. Haan (1970), On regular variation and its application to the weak convergence of sample extremes. Mathematical Centre Tract 32, Amsterdam. 
Risk Analysis and Crisis Response in Big Data Era (RAC-16)

[8] R.L. Smith (1987), Estimating tails of probability distributions. The Annals of Statistics, 15(3):1174-1207.

[9] A.J. McNeil (1999), Extreme value theory for risk managers. Internal Modeling CADII, Risk Books, 93-113.

[10] A.J. McNeil, Frey (2000), Estimation of Tail-related Risk measures for Heteroscedastic Financial Time series: An Extreme Value Approach. JEF., 7:271-300

[11] J. Pickands (1975), Statistical inference using extreme value order statistics. Annals of Statistics, 3:119-131

[12] J Beiriant, JL. Teugels, P. Vynckier (1996), Practical Analysis of Extreme values. Leuven University Press

[13] 田宏伟,詹原瑞.极值理论（EVT）方法用于受 险价值(VaR)计算中的实证比较与分析.系统 工程学报,2000（10）;27-36

[14] 王元月等.國值选取的 hill 估计方法改进一基 于极值理论中 POT 模型的实证. 中国海洋大学 学报,2012 (3):42-46

[15] 周开国.应用极值理论计算在险价值（VaR） 一对恒生指数的实证分析. 预测, 2002, 21 (3) :37-41

[16] 欧阳资生. 厚尾分布的极值分位数估计与极值 风险测度研究. 数理统计与管理,2008 (1) $: 70-75$

[17] 杨刚等.Hall 分布族在巨额索赔数据的极值 分位数估计与风险度量中的应用.系统工程学 报,2008（7）:112-116

[18] 肖海清,孟生旺.极值理论及其在巨灾再保险 定价中的应用数理统计与管理,2013 (3) :240-246

[19] 刘鹃, 李永.中国地震损失分布与巨灾债券定 价研究.财贸研究,2009 (6) :82-88

[20] 卓志等.巨灾风险厚尾分布:POT 模型及其应 用 [J],保险研究 2011（8）:13-19

[21] 钱小仕等.广义极值分布在地震危险性分析中 的应用.地震研究,2012 (1) :73-77

[22] Chongfu Huang (2011), A Discrete Model of the Expected Loss for Catastrophe Insurance in Natural Disasters. Journal of Risk Analysis and
Crisis Response, 1(1): 48-58

[23] Chongfu Huang (2011),Internet of Intelligences in Risk Analysis for Online Services . Journal of Risk Analysis and Crisis Response, 1(2): 110-117

[24] G. Chichilnisky (2000), An axiomatic approach to choice under uncertainty with catastrophic risks. Resource and Energy Economics 22:221-231

[25] K,Hansson, M.Danielson and L. Ekenberg (2008),A framework for evaluation of flood management strategies. Journal of Environmental Management,86:465-480

[26] GAO (2002) , Statement of Richard J.Hillman. General Accounting Office. Terrorism Insurance Rising Uninsured Exposure to Attacks Heightens Potential Economic Vulnerabilities.US Govt. General Accounting Office, February 27, 2002

[27] Mohamed Gad-el-Hak (2008), Large-scale disasters prediction, control and mitigation. Cambridge University Press, New York, USA, 2008: 1-4

[28] Swiss Re. (2009), World insurance in 2008: life Premiums fall in the industrialized countries, strong growth in the emerging economies.

[29] 马宗晋等.自然灾害与减灾 $[\mathrm{M}]$. 北京:地震出 版社, 1990

[30] 汤爱平等:自然灾害的概念、等级.自然灾害学 报,1999（8）:61-65

[31] 李军,李德志.论巨灾风险证券化与风险融资 制度的发展.特区经济,2005(8):89-90

[32] 邓国取,浅析巨灾及其实证分析:以农业巨灾 定义为例.管理观察,2009(1):32-33

[33] 马宗晋,对我国区域减灾的建议.中国减 灾,2010(1):30

[34] 史培军等,全球环境变化与综合灾害风险防范 研究.地球科学进展 2009,24（4）:428-435 